\title{
Eosinophilic Esophagitis in Refractory GERD
}

\author{
Mostafa Elshami \\ Tropical Medicine Department, Faculty of Medicine, Zagazig University, Egypt \\ See article pages:59-68
}

\section{Background}

Eosinophilic esophagitis (EE) is a disease in which upper intestinal symptoms is associated with dens eosinophilic infiltration of squamous epithelium or deeper eosophageal tissue. Neither symptoms nor eosinophilia respond to treatment with proton pump inhibitors (PPI), but it responds well to topical steroids. The pathophysiological mechanisms are likely related to allergic inflammation, not to underlying motility disorder as in gastroesophageal reflux disease (GERD) [1].

Diagnosis of EE is made by esophageal biopsy during endoscopic examination with the presence of eosinophiles (>15 per HPF) in the tissue with or without peripheral eosinophilia. There is an overlap between EE and refractory GERD as regard tissue eosinophils as refractory GERD patients may have eosinophilic infiltration in esophageal biopsy but with counts less than 15 per HPF [2]. The etiology of EE is poorly understood, allergic response has been implicated. The offending agent of this allergic reaction may be ingested or inhaled allergen as proved by skin hypersensitivity test. In EE, Th2- type immune response is present with increased level of eosinophilic active Th2 cytokines, as IL-4, IL-5, IL-13 [3].

$\mathrm{EE}$ is an uncommon disease and there are insufficient studies to know its exact prevalence and incidence, it is common in males than females, in children more than adults, it must be considered in patients with refractory GERD [4].

\section{Summary of the paper}

The paper entitled "Eosinophilic Esophagitis in Patients with Refractory Gastro-esophageal Reflux Disease (GERD) ". The authors enrolled 100 patients with GERD ; 50 patients of them have refractory GERD aiming to evaluate the frequency of $\mathrm{EE}$ in patients with refractory GERD and, the clinical and pathological differences between both groups. They performed basic investigations together with endoscopy with esophageal biopsy, esophageal manometry, 24-hour PH metry, pathological and immunohistochemical examination of the biopsy specimen. They found that the frequency of EE is $4 \%$ in patients with refractory GERD and tissue eosinophilia is significantly increased in refractory GERD patients while peripheral eosinophilia was the same. They also stated that pathological examination with ordinary stains gave the same results as immunohistochemical staining.

\section{Comment on the study}

The frequency of EE is not exactly known but the frequency in special population groups is suggested in clinical trials, but the number of patients in this study was too small to achieve statistical significance, but the results support the role of EE in the differential diagnosis of refractory GERD. The paper clarify the difference between GERD and refractory GERD without stress on EE patients as regard clinical findings this is because of little number of patients. The authors describe that rings and furrows are the most common endoscopic findings in $\mathrm{EE}$ patients while these findings were present in only 2 patients.

\section{Recommendations:}

Large population sample is required to better determination of $\mathrm{EE}$ frequency, and proper understanding the clinical, laboratory and endoscopic data of this disease. Better understanding of clinical outcomes, complications and response to treatment.

\section{References}

1- Fox VL, Nurko S , Furuta GT. Eosinophilc eosophagitis, it is not just kid's stuff. Gastroenterointest Endoscopy 2002; 56: 260-270.

2- Mishra A, Hogan SP, Lee JJ. Fundamental signals that regulate eosinophil homing to the gastrointestinal tract. J Clin Invest 1999; 103:1719-1727. 
3- Blanchard C, Wang N, Stringer. Eotaxin-3 and uniquely conserved gene-expression profile in eosinophiliv eosophagitis. J Clin Invest 2006; 116 : 536-547.
4- Potter JW, Saiean K, Staff D. Eosinophilic eosophagitis in adults: an emerging problem with unique eosophageal features. Gastrointest Endoscopy 2004; 59 : 355-361. 